\title{
YELLOW FEVER AND VACCINATION
}

It seems to be the opinion of the consensus that yellow fever represents a serious threat to the population for our country. That is what may be supposed from the far-reaching repercussions caused, as much among specialists as among the lay population, on the occasion of the publication, in the recent past, of the news which reported the presence of the mosquito Aedes aegypti in various parts of the Brazil territory. The lay news referred to this mosquito in such a way as simply to confuse it with the in fection itself.

Even though there was no urban case, the origin of which could be imputed to the action of that vector, there can be no doubt that the possibility always exists. This is so because it is impossible to ignore the fact of the existence of the yellow fever Flavivirus in Brazil, even though, until now, limited to the forest environment. In view of this the occurrence of human cases in urban zones in fected by the Aedes aegypti, despite its forest origin, came to be considered a real risk, in view of the outbreaks possibility in the urban environment. The public services, both federal and state, parallel to the combat against the urban vector, undertook a wide-reaching campaign of vaccination in those areas considered to be at risk, that is, those adjacent to the great forest regions of northem and central Brazil, susceptible to receiving and harboring the cases which should arrive with the viral agent in the circulating blood.

Among the reflections which such facts raise, it is that regarding the immunization of the exposed population by means of the mass application of the available vaccine, known to be efficient. These thoughts, when they touch on a subject of as a polemic nature, arise again when one hints at the possibility of including this vaccination as a routine practice. Leaving aside states of emergency which would justify this mass vaccination, one must weigh up various aspects. In a recent publication the World Health Organization (WHO, 1986), in dealing with the prevention and control of yellow fever in Africa, emphasizes certain basic principles which, mutatis mutandis, could provide information applicable to the Americas in general and to Brazil in particular.

For the formulation of a strategy with a view to prevention, on the medium and long term, it is necessary to establish clearly defined objectives. These would include the delimitation of the endemic areas, the implantation of a system of epidemiological surveillance, the prior establishment of plans for emergency situations, the implementation of mass preventive immunization for the groups at risk and the control of the vector mosquitoes whenever possible. Clearly the putting to practical use of all the favorable conditions and the identification of all the obstacles to be overcome is inherent in this strategy. It is still clearer that there is the need for the existence of an undertaking, in terms of health policy, at national level, in order to guarantee the resources for these activities.

Within this context one may relevantly include the attitude to be adopted in the policy of immunization at the level of the population. Until recently, mass vaccination against yellow fever was prescribed, limitedly, as an emergency measure in epidemic situations. Such limitation is, however, being set aside and one increasingly observes changes in orientation in the sense that several countries have begun routine immunization programs on the basis of the expectation that one single vaccination could result in lasting protection. Thus, ideally, a whole population might be protected, and this state of immunity would be maintained by the routine vaccination of the infant and immigrant contingents. In this way, only a lack of resources could justify the limitation of immunization to the population at risk and to occasions of outbreaks of the virus disease, or in the face of the extreme likelihood of such events. There is therefore no doubt, judging from the arguments of the experts who undersigned the publication mentioned, that the tendency in vaccination against yellow fever is to include it in the routine of infant immunization, as from 9 months of age, so as to coincide with the immunization against measles as already practised.

In the light of these recent practices in Public Health, even though apparently limited to the African continent, one cannot avoid wondering what the best course for a country like Brazil would be. On the occasion of the wave of sensationalism which colored the lay news on the presence of Aedes aegypti, some experts emitted opinions contrary to mass vaccination. However, apart from these episodes, it is time the reasons which could lead to routine anti-yellow fever vaccination in our national territory were pondered. The first one is, without doubt, the existence of the circulating viral agent. Although it is restricted to the primitive forest environment, there is no way of denying the possibility of its migration to the modified areas where the presence of residual forests suggests the possibility of harboring those elements involved in the zoonotic cycle, such as the occurrence of Haemagogus mosquitoes. The second is, incontestably, the presence of Aedes aegypti in the urban environment, even that of large cities such as S.Paulo. Allow me to 
doubt the practical possibilities of its erradication in the way it was supposed to have occurred some decades age. The present stage of urbanization, living habits, population density, the variety of recipients at present used, the means of transport, as well as other aspects, are totally different from those that were to be observed at that time. Finally, there is no lack of mosquito species with vectorial possibilities which may be in process of adaptation to the artificial environment. These culicidae, such as the Aedes scapularis for example, might be capable of establishing a link between the forest and domiciliary environments, in as or more efficacious a way that of the circulation of human cases with viremia.

Thus here there is something which is deserving of the attention of our health authorities - i.e., the study of the possibility of the adoption of routine vaccination against yellow fever. At the very least as a means to stimulate the search for a solution of the problem in a comprehensive way, not in a way that is simply centred on some aspects of its control at present known to us.

\section{REFERENCES}

WORLD HEALTH ORGANIZATION. Prevention and control of yellow fever in Africa. Geneva, 1986.

Oswaldo Paulo Forattini Department of Epidemiology of "Faculdade de Saude Pública / USP" 